\title{
Anatomical and Pathological Findings of Magnetic Resonance Imaging in Idiopathic Sudden Sensorineural Hearing Loss
}

\author{
Min Bum Kim ${ }^{1,2}$, Jihyun $\mathrm{Lim}^{3}$, and II Joon Moon ${ }^{1,2}$ \\ ${ }^{1}$ Department of Otorhinolaryngology-Head and Neck Surgery, Samsung Medical Center, School of Medicine, Sungkyunkwan University, \\ Seoul, Korea \\ ${ }^{2}$ Hearing Research Laboratory, Samsung Medical Center, Seoul, Korea \\ ${ }^{3}$ Center for Clinical Epidemiology, Samsung Medical Center, Seoul, Korea
}

\begin{abstract}
Received April 14, 2020
Revised June 2, 2020

Accepted July 27, 2020
\end{abstract}

Background and Objectives: We sought to evaluate the diagnostic and prognostic value of measurable parameters of internal auditory canal (IAC) magnetic resonance imaging (MRI) in patients with idiopathic sudden sensorineural hearing loss (ISSNHL). Subjects and Methods: We retrospectively reviewed the patients with ISSNHL who underwent IAC MRI from January 2008 to March 2019. Measurable parameters of IAC MRI, such as the diameter of the IAC, bony cochlear nerve canal, and cross-sectional area of the cochlear nerve, were measured by a single examiner. These parameters were then compared between the affected and healthy sides. Inner-ear abnormalities such as intralabyrinthine hemorrhage or labyrinthitis were also evaluated. The relationship between the surveyed parameters and the diagnosis of ISSNHL was assessed. Results: A total of 208 patients with ISSNHL were included. The measured parameters of IAC MRI were not different between the affected and healthy sides and were also not associated with the diagnosis of ISSNHL. However, inner-ear abnormalities of IAC MRI in ISSNHL displayed a significant association with worse hearing before and after treatment. An age that was older than 40 years also correlated with poorer outcomes. Further, inner-ear abnormalities were more frequently detected when IAC MRI was performed early after ISSNHL onset. Conclusions: Patients with ISSNHL and inner ear abnormalities such as intralabyrinthine hemorrhage or labyrinthitis identified via IAC MRI may experience poorer hearing outcomes. To detect such abnormal findings, it is recommended to perform IAC MRI early after the onset of ISSNHL.

J Audiol Otol 2020;24(4):198-203
KEY WORDS: Hearing loss · Sudden hearing loss · Magnetic resonance imaging · Labyrinth diseases · Labyrinthitis.

\section{Introduction}

Sudden sensorineural hearing loss (SSNHL) is a disorder defined as a subset of hearing loss that is sensorineural in nature and that occurs within a 72-hour period. The audiometric criterion for SSNHL is a decrease in the hearing of at least 30 decibels $(\mathrm{dB})$ that affects at least three consecutive frequencies. The incidence of SSNHL is five to 27 per 100,000 people

This is an Open Access article distributed under the terms of the Creative Commons Attribution Non-Commercial License (https://creativecommons.org/licenses/by-nc/4.0/) which permits unrestricted non-commercial use, distribution, and reproduction in any medium, provided the original work is properly cited. annually [1]. About $90 \%$ of patients with SSNHL present no identifiable cause of the hearing loss; this condition is defined as idiopathic sudden sensorineural hearing loss (ISSNHL) [1].

There are several hypothetical causes predisposing a patient to ISSNHL, such as micro-circulation disorders or inflammation such as that experienced in conjunction with a viral infection or autoimmune disease [2]. However, the exact cause of ISSNHL remains largely unknown, resulting in a lack of universally agreed-upon treatment protocols. Corticosteroids have been considered as the mainstream treatment option in the past; however, guidelines now suggest that the initial use of corticosteroids as a treatment modality for ISSNHL is not strongly 
recommended [1]. These guidelines also contend that hyperbaric oxygen therapy may be a reasonable option for solving the hypoxic problems that occur in the inner ear [1]. Meanwhile, other pharmacologic therapies are strongly suggested to be contraindicated. The recovery rate from ISSNHL has been reported to range between 45 and $66 \%$ with standard corticosteroid treatment [3].

Magnetic resonance imaging (MRI) of the internal auditory canal (IAC) in patients with SSNHL is usually recommended to evaluate the retrocochlear pathology such as the presence of vestibular schwannoma (VS) or cerebellopontine angle tumor. The prevalence of VS in patients with SSNHL was reported as $3 \%$ in a recent study [4]. Some research has postulated that, if intralabyrinthine hemorrhage is identified on MRI, the prognosis for hearing restoration in patients with SSNHL is poorer [5]. However, the understanding of the value of MRI is more limited when no such lesion exists.

With improved MRI technologies, we can measure the overall IAC diameter as well as those of tiny structures within the IAC. Several studies have reported on differences in the size of the cochlear nerve $(\mathrm{CN})$ in normal and hearing-impaired individuals [6-8]. However, previous reports on the size of the $\mathrm{CN}$ or the diameter of the IAC in patients with ISSNHL are lacking. In this study, we measured those parameters and analyzed the results of IAC MRI in patients with ISSNHL to evaluate the diagnostic and prognostic values of the imaging test.

\section{Subjects and Methods}

\section{Subjects}

We retrospectively reviewed the patients who underwent IAC MRI from January 2008 to March 2019. Patients diagnosed with other disorders such as VS, Meniere's disease (MD), or conductive hearing loss were excluded from this investigation. Patients with bilateral SSNHL and $<18$ years were also excluded.

All of the patients enrolled in our study had visited Department of Otorhinolaryngology-Head and Neck Surgery, Samsung Medical Center within one month after the onset of sudden hearing loss. At the time of the first visit, the diagnosis of SSNHL was made based on pure tone audiometry results. The audiometric criterion for SSNHL was hearing loss of at least $30 \mathrm{~dB}$ on pure tone average (PTA) occurring in at least three consecutive frequencies. Because some patients could not visit Department of Otorhinolaryngology-Head and Neck Surgery, Samsung Medical Center within 72 hours of symptom onset due to our tertiary hospital system status, we included patients diagnosed with SSNHL at other primary or secondary hospitals with suitable PTA results for the disorder.

\section{MRI protocol}

MRI was performed with T2-weighted turbo spin-echo (TSE) axial images of the entire brain [repetition time (TR), 2,000 ms; echo time (TE), $30 \mathrm{~ms}$; section thickness, $2 \mathrm{~mm}$; slice spacing, $2 \mathrm{~mm}$; field of view, $19 \mathrm{~cm}$; acquisition time, 338 seconds]. Oblique sagittal images of the IAC were reformed by the multiplanar reconstruction procedure.

\section{Measurement}

On oblique sagittal images of IAC MRI, we measured the horizontal and vertical widths of the $\mathrm{CN}$ and facial nerve (FN) on each side (Fig. 1). Oblique sagittal images were selected in the middle of the IAC where the $\mathrm{CN}, \mathrm{FN}$, and vestibular nerves were individually identifiable. The cross-sectional area (CSA) was calculated $[\pi(\mathrm{V} / 2)(\mathrm{H} / 2)]$ for the $\mathrm{CN}$ and $\mathrm{FN}$, respectively. The diameters of the fundus and porus of the IAC and the bony cochlear nerve canal (BCNC) were also measured on axial T2-weighted TSE IAC MRI scans. The diameter of the IAC was calculated using the axial image in which the ipsilateral lateral semicircular canal was totally visible (Fig. 2). The width of the BCNC is usually measured via temporal bone computed tomography (TBCT); thus, we compared the measured width of the BCNC on IAC MRI and TBCT scans in some patients (Fig. 3). All measuring was performed by an otologist (M.B.K) to all clinical information. Formal reading of the MRI by a head and neck radiology specialist was also completed to collect abnormal findings in the inner ear.

\section{Treatment protocol and outcome evaluation}

All patients with no contraindications were treated with high-dose systemic steroid therapy. Most of the patients received per-oral methylprednisolone $(1 \mathrm{mg} / \mathrm{kg} /$ day up to $60 \mathrm{mg}$

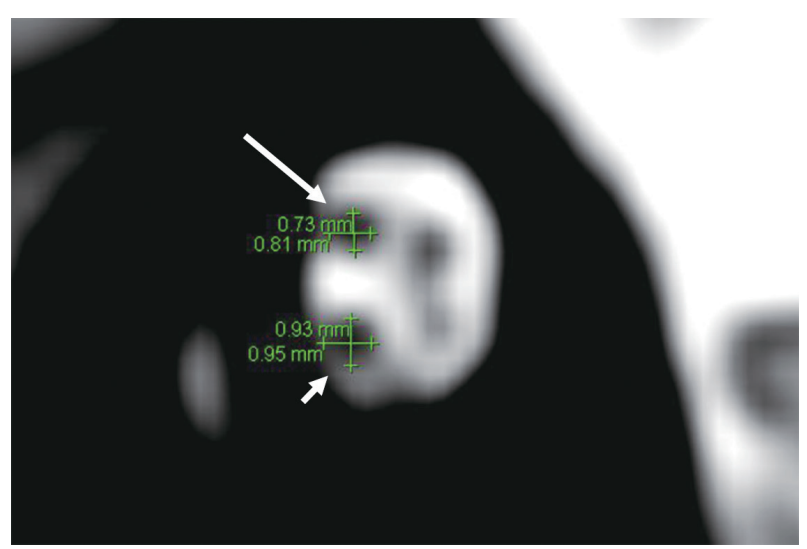

Fig. 1. Measuring the horizontal and vertical widths of the cochlear nerve (CN) (long arrow) and facial nerve (FN) (short arrow) of each side on oblique sagittal images of internal auditory canal (IAC) magnetic resonance imaging. Oblique sagittal images were selected from the middle of the IAC where the cochlear nerve, FN, and vestibular nerves could be individually identified. 
per day for seven days, with five days of tapering), though some received intravenous dexamethasone $(10 \mathrm{mg} /$ day with tapering). Fifty-eight (27.9\%) of the 208 study participants had
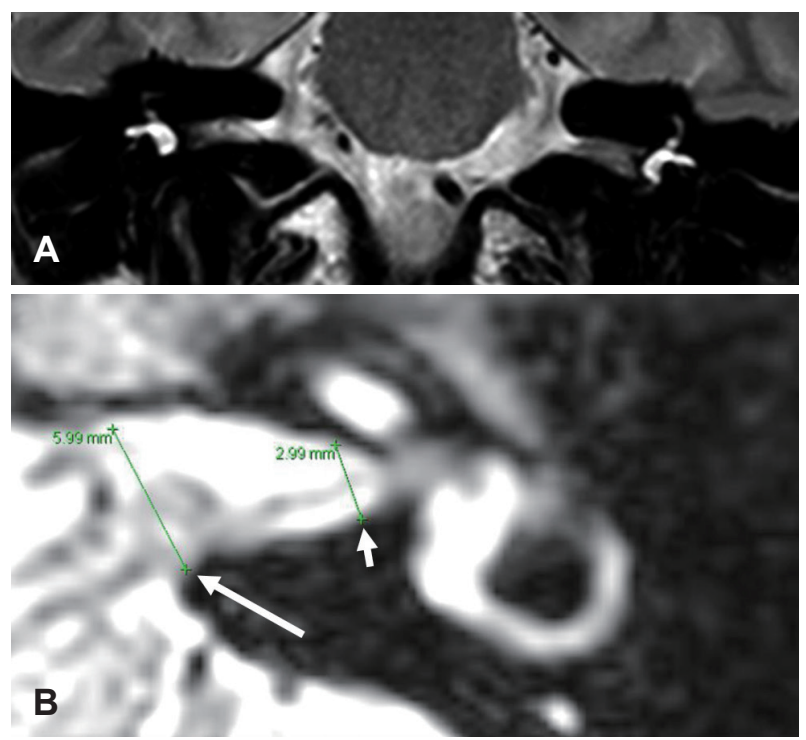

Fig. 2. Measuring the anteroposterior diameter of the fundus (short arrow) and porus (long arrow) of the internal auditory canal (IAC) on axial T2-weighted magnetic resonance imaging scans. The IAC was placed at the same vertical level as the lateral semicircular canal (A); thus, the diameter of the IAC was measured on the axial image, where the ipsilateral lateral semicircular canal was totally visible (B).
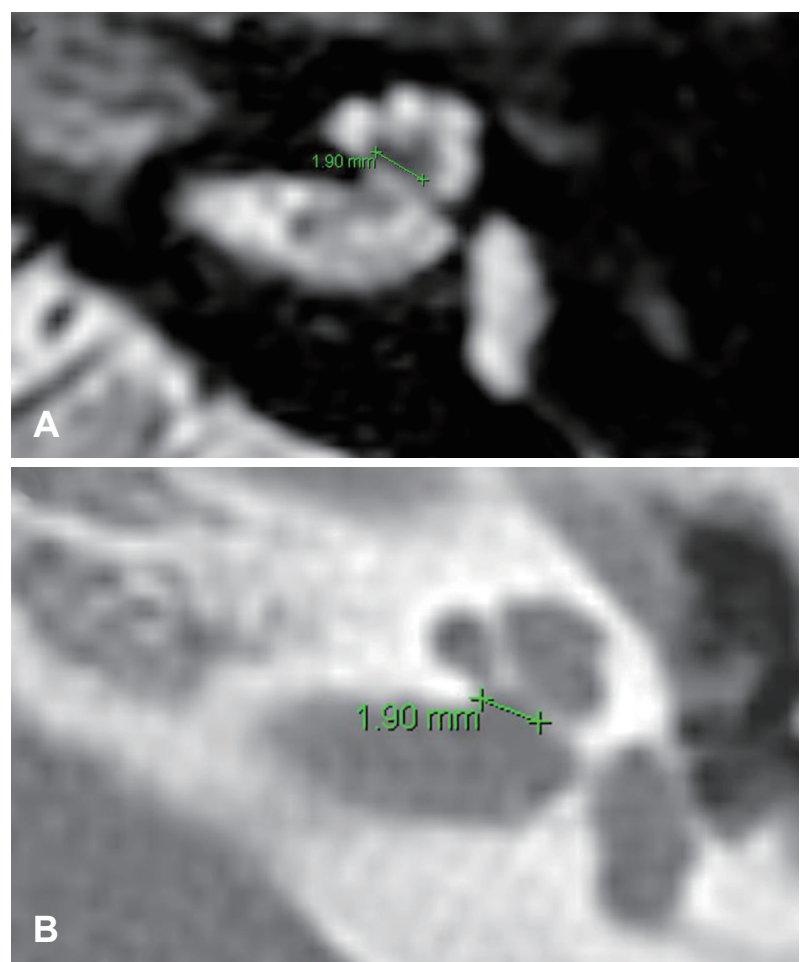

Fig. 3. Measuring the width of the bony cochlear nerve canal on an internal auditory canal magnetic resonance imaging scan (A) and high-resolution temporal bone computed tomography scan of the same patient $(B)$. been primarily treated at other hospitals and one did not receive high-dose steroid therapy due to a previous history of side effects from steroid use. Therefore, 147 (70.7\%) of the 208 enrolled patients were treated with high-dose systemic steroid therapy at Samsung Medical Center. For patients who experienced no or partial recovery with high-dose systemic steroid therapy, intratympanic steroid injection was given as a salvage treatment. Ninety-nine (47.6\%) of the study participants were injected with intratympanic steroids at least once.

PTA performed at the first and last visits were analyzed to evaluate the treatment outcome. The evaluation of treatment outcome followed the criteria previously used by Ho, et al. [9]. Complete recovery was defined as an average threshold across 250, 500, 1,000, 2,000, and 4,000 Hz within $25 \mathrm{~dB} \mathrm{HL}$; marked improvement was defined as a hearing gain of more than 30 $\mathrm{dB}$ in the average threshold; slight improvement was defined as a hearing gain of between $10 \mathrm{~dB}$ and $30 \mathrm{~dB}$ in the average threshold; and nonrecovery was defined as an average hearing gain within $10 \mathrm{~dB}$ (Table 1).

\section{Statistical analysis}

We conducted all statistical analysis using SAS version 9.4 (SAS Institute Inc., Cary, NC, USA). A paired t-test was used to compare the measured values sourced from IAC MRI between the affected and healthy sides. The relationships between hearing outcomes and different variables were analyzed with ordinal logistic regression. A $p$-value less than 0.05 was considered to be statistically significant.

\section{Ethical approval}

All procedures performed in studies involving human participants were in accordance with the ethical standards of the institutional and/or national research committee [Samsung Medical Center (IRB No. 2019-11-002-001)] and with the 2013 Helsinki declaration and its later amendments or comparable ethical standards.

\section{Results}

Two hundred eight patients with ISSNHL were included in

Table 1. Classification of hearing outcomes after treatment of idiopathic sudden sensorineural hearing loss

\begin{tabular}{|c|c|}
\hline Classification & Definition \\
\hline Complete recovery & PTA* $\leq 25 \mathrm{~dB}$ HL after treatment \\
\hline Marked improvement & PTA* improvement $\geq 30 \mathrm{~dB} \mathrm{HL}$ \\
\hline Slight improvement & $10 \mathrm{~dB} \mathrm{HL} \leq$ PTA* improvement $<30 \mathrm{~dB} \mathrm{HL}$ \\
\hline No recovery & PTA* improvement $<10 \mathrm{~dB} \mathrm{HL}$ \\
\hline
\end{tabular}

*pure tone average (PTA) of $0.5,1,2$, and $4 \mathrm{kHz}$ 
accordance with the inclusion criteria. The average age of the enrolled patients was $52.23 \pm 13.45$ years (range: $18-81$ years) with 103 females (49.5\%) and 105 males. One hundred ten patients $(52.9 \%)$ had left-sided hearing loss, the others showed right-sided hearing loss. The mean follow-up duration was 397 days and the median value of the follow-up duration was 87 days. Some patients received PTA long after the end of treatment when they visited the hospital to undergo MRI.

Measured data from IAC MRI, such as the CSA of the CN, $\mathrm{BCNC}$, and diameter of the IAC, all displayed normal distributions. Oblique sagittal images were collected from only 60 patients; the CSA of the $\mathrm{CN}$ was measured in those individuals. The other measured values were gathered from all 208 patients. All measured values showed tendencies to be lower on the affected side, but the differences were not statistically significant (Table 2).

MRI confirmed not only the above measurements but also abnormal findings of the inner ear such as increased signal intensity or contrast enhancement of the inner ear. There were 30 cases (14.4\%) with abnormal findings and 178 cases without abnormal findings. We analyzed the relationship between the above measurements, the presence of an abnormality in the inner ear, and previously known prognostic factors such as age and presence of dizziness.

The hearing outcome revealed that 32 cases $(21.8 \%)$ experienced complete recovery, 16 cases $(10.9 \%)$ showed marked recovery, 29 cases (19.7\%) had slight recovery, and 70 (47.6\%) patients displayed no improvement. These results did not meet the general SSNHL prognosis standard. Patients older than 40 years of age didn't experience a significant difference relative to younger patients in terms of the hearing threshold initially ( $p=0.21$ ) but showed a 3.27-fold higher tendency toward hearing deterioration. The presence of dizziness had a relationship with worse hearing initially ( $p=0.02$ ) but did not affect the hearing outcome in this study (Table 3). MRI measurements also presented no correlation with the prognosis, but the hearing

Table 2. Average of measured values on the affected and healthy sides

\begin{tabular}{lrrrr}
\hline \multirow{2}{*}{ Variable } & \multirow{2}{*}{$\mathrm{n}$} & \multicolumn{2}{c}{ Side } & $\mathrm{p}$ - \\
\cline { 3 - 4 } & & Healthy & Affected & value \\
\hline CN CSA & 60 & $3.14(0.52) \mathrm{mm}^{2}$ & $3.03(0.44) \mathrm{mm}^{2}$ & 0.14 \\
BCNC diameter & 208 & $2.04(0.28) \mathrm{mm}$ & $2.03(0.27) \mathrm{mm}$ & 0.14 \\
IAC diameter & & & & \\
IAC_F & 208 & $3.64(0.51) \mathrm{mm}$ & $3.61(0.49) \mathrm{mm}$ & 0.39 \\
IAC_P & 208 & $5.98(1.11) \mathrm{mm}$ & $5.96(1.06) \mathrm{mm}$ & 0.71 \\
\hline
\end{tabular}

Values are presented as mean (standard deviation). $\mathrm{CN}$ : $\mathrm{CO}-$ chlear nerve, CSA: cross-sectional area, BCNC: bony cochlear nerve canal, IAC: internal auditory canal, IAC_F: fundus of the IAC, IAC_P: porus of the IAC restoration prognosis was worse in conjunction with the presence of abnormalities in the inner ear in the other cases (Fig. 4).

Time intervals between the onset of ISSNHL and IAC MRI conduct varied and we analyzed the correlation between findings of the inner ear and the time interval between symptom onset and MRI performance. Interestingly, those patients who underwent MRI within two weeks of ISSNHL onset showed higher rates of inner ear abnormality discovery than did those patients who underwent MRI at two weeks from onset (Table 4).

\section{Discussion}

The recent guidelines of the American Academy of Otolar-

Table 3. Odd ratios of each variable in relation to worse hearing outcomes

\begin{tabular}{lccc}
\hline \multicolumn{1}{c}{ Variable } & OR & $95 \% \mathrm{Cl}$ & p-value \\
\hline CN CSA & 0.57 & $0.15-2.15$ & 0.41 \\
BCNC diameter & 2.41 & $0.76-7.57$ & 0.13 \\
IAC diameter & & & \\
IAC_F & 0.74 & $0.40-1.39$ & 0.35 \\
IAC_P & 1.18 & $0.88-1.58$ & 0.28 \\
Age (years) & & & \\
Age $<40$ & & Reference & \\
Age $\geq 40$ & 3.27 & $1.40-7.62$ & $<0.01$ \\
Dizziness & & & \\
Absent & & Reference & \\
Present & 1.48 & $0.77-2.86$ & 0.24 \\
\hline OR:
\end{tabular}

OR: odds ratio, $\mathrm{Cl}$ : confidence interval, $\mathrm{CN}$ : cochlear nerve, CSA: cross-sectional area, BCNC: bony cochlear nerve canal, IAC: internal auditory canal, IAC_F: fundus of the IAC, IAC_P: porus of the IAC

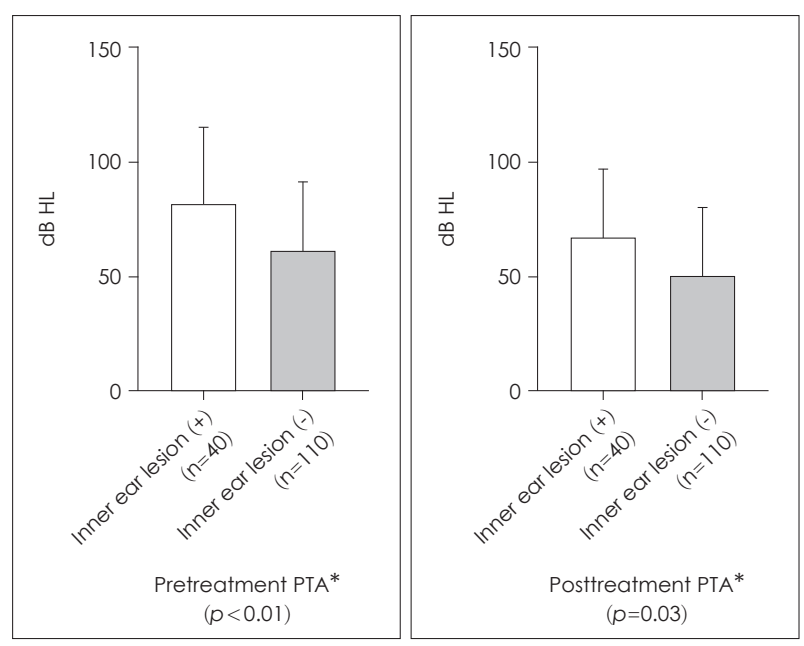

Fig. 4. Average pure tone threshold of patients with or without inner-ear lesions on internal auditory canal magnetic resonance imaging scans. Patients with inner-ear lesions experienced significantly worse hearing than those without inner-ear lesions both preand posttreatment. *pure tone average (PTA) of $0.5,1,2$, and $4 \mathrm{kHz}$. 
Table 4. Correlation between time interval from the onset of hearing loss to magnetic resonance imaging of the internal auditory canal and the detection of inner-ear lesions

\begin{tabular}{lccc}
\hline \multicolumn{1}{c}{ Variable } & OR & $95 \% \mathrm{Cl}$ & $p$-value \\
\hline Within 14 days & \multicolumn{5}{c}{ Reference } \\
Over 14 days & 0.25 & $0.11-0.58$ & $<0.01$ \\
\hline OR:
\end{tabular}

OR: odds ratio, Cl: confidence interval

yngology - Head and Neck Surgery (AAO-HNS) recommend that patients with SSNHL be evaluated for retrocochlear pathologies using IAC MRI or auditory brainstem response (ABR) assessments [1]. MRI is more cost-effective than ABR to use to differentiate retrocochlear lesions such as VS. [10]; thus, IAC MRI is considered to be the gold standard test for detecting VS. The prevalence of VS within SSNHL patients is reported to be $3 \%$ [4].

Recent advances in MRI techniques have enabled more accurate assessments of labyrinthine lesions in SSNHL [5]. Especially, using fluid-attenuated inversion recovery (FLAIR) MRI, we can identify underlying pathologies in ISSNHL cases that were previously designated as being idiopathic. FLAIR MRI can assist clinicians in detecting increased protein concentrations or hemorrhage in the labyrinth that can contribute to hearing loss [11].

FLAIR MRI is a T2weighted sequence that incorporates an inverted signal of the cerebrospinal, endolymph, and perilymph fluids. Therefore, FLAIR MRI sequences can be used to identify methemoglobin in the cerebrospinal fluid in addition to revealing elevated protein and cellular concentrations. On FLAIR images, the hypersignal intensity in the labyrinth may reflect the presence of blood and protein within the inner ear [11].

Also, recent improvements in the resolution of MRI can facilitate the identification of facial, cochlear, and superior and inferior vestibular nerves and distinguish each of these on oblique sagittal images of IACs. Several previous studies have reported results of the diameters and CSAs of CNs and FNs in normal or hearing-impaired populations [6-8,12].

In a recent study, the CSAs of CNs calculated from measured horizontal and vertical diameters were $0.77 \mathrm{~mm}^{2}$ for the affected side and $0.78 \mathrm{~mm}^{2}$ for the healthy side, indicating no difference existed between the two sides. Our calculated average value was relatively low as compared with the results of previously reported studies $[7,12]$ but our data also indicated a normal distribution existed. Differing results may therefore be due to variations in MRI protocol details. Some previous studies reported that the size of CNs was reduced in patients with sensorineural hearing loss or deafness [6,8]. Our study evaluated patients with SSNHL whose duration of hearing loss was relatively short. We considered this to be the reason for why our data showed no difference between the sizes of $\mathrm{CNs}$ in af- fected and healthy sides, respectively. Other measured values such as the diameter of the IAC and BCNC similarly displayed no difference between the two sides.

As previously shown $[11,13,14]$, labyrinthine lesions such as intralabyrinthine hemorrhage were associated with worse hearing with or without treatment in our study. Labyrinthine lesions on IAC MRI should be considered to be an important prognostic factor for SSNHL. The possibility of detecting such labyrinthine lesions on IAC MRI is significantly higher when the image is taken within two weeks after onset. This can be explained by the course of hemoglobin decomposition: specifically, the hemoglobin in hemorrhage is metabolized from oxyhemoglobin to deoxyhemoglobin, methemoglobin, and then hemosiderin. Thus, after two weeks, the hemoglobin has become hemosiderin which produces a hyposignal intensity on FLAIR images [11]. Thus, we recommend conducting IAC MRI within two weeks of SSNHL onset to detect not only chronic retrocochlear lesions but also acute or subacute labyrinthine lesions. This may assist clinicians in better predicting a patient's prognosis.

The recovery rate from ISSNHL is accepted as being up to two-thirds of cases when using high-dose systemic steroid treatment [1]. In our study, the proportion of patients who experienced complete or marked recovery was less than one-third, which is only half of the expected rate even when including the slightly recovered group. Not all patients with SSNHL underwent MRI and those with intractable hearing loss after initial treatment tended to be included in the group who underwent imaging. This may be the reason for the worse hearing outcomes in our SSNHL sample.

This study had some limitations. IAC MRI was not performed on all patients with SSNHL and oblique sagittal images were obtained from only some of the patients. Some patients had started treatment at local clinics so the evaluation of hearing outcome was incomplete. However, we measured the size of CNs or IACs on IAC MRI scans of a large number of patients with ISSNHL in whom other etiologies such as VS and MD were excluded. We also analyzed the correlation between the time of MRI after onset and the results. This study is expected to support clinicians in evaluating the prognosis of and counseling patients with ISSNHL.

\section{Acknowledgments \\ None}

\section{Conflicts of interest}

The authors have no financial conflicts of interest.

\section{Author Contributions}

Conceptualization: Il Joon Moon. Data curation: Min Bum Kim. Formal analysis: Jihyun Lim. Investigation: Min Bum Kim. Method- 
ology: Min Bum Kim and Il Joon Moon. Project administration: Il Joon Moon. Supervision: Il Joon Moon. Validation: Il Joon Moon. Visualization: Min Bum Kim and Jihyun Lim. Writing — original draft: Min Bum Kim and Il Joon Moon. Writing—review \& editing: Min Bum Kim. Approval of final manuscript: All authors.

\section{ORCID iDs}

Min Bum Kim

Jihyun Lim

Il Joon Moon

https://orcid.org/0000-0001-5418-5176

https://orcid.org/0000-0002-0318-9334

https://orcid.org/0000-0002-3613-0734

\section{REFERENCES}

1) Chandrasekhar SS, Tsai Do BS, Schwartz SR, Bontempo LJ, Faucett EA, Finestone SA, et al. Clinical practice guideline: sudden hearing loss (update). Otolaryngol Head Neck Surg 2019;161(1_suppl):S1-45.

2) Ban JH, Jin SM. A clinical analysis of psychogenic sudden deafness. Otolaryngol Head Neck Surg 2006;134:970-4.

3) Byl FM Jr. Sudden hearing loss: eight years' experience and suggested prognostic table. Laryngoscope 1984;94(5 Pt 1):647-61.

4) Fujita T, Saito K, Kashiwagi N, Sato M, Seo T, Doi K. The prevalence of vestibular schwannoma among patients treated as sudden sensorineural hearing loss. Auris Nasus Larynx 2019;46:78-82.

5) Cho J, Cheon H, Park JH, Lee HJ, Kim HJ, Choi HG, et al. Sudden sensorineural hearing loss associated with inner ear lesions detected by magnetic resonance imaging. PLoS One 2017;12:e0186038.

6) Herman B, Angeli S. Differences in cochlear nerve cross-sectional area between normal hearing and postlingually deafened patients on MRI. Otolaryngol Head Neck Surg 2011;144:64-6.

7) Kang WS, Hyun SM, Lim HK, Shim BS, Cho JH, Lee KS. Norma-

tive diameters and effects of aging on the cochlear and facial nerves in normal-hearing Korean ears using 3.0-tesla magnetic resonance imaging. Laryngoscope 2012;122:1109-14.

8) Sildiroglu O, Cincik H, Sonmez G, Ozturk E, Mutlu H, Gocgeldi E, et al. Evaluation of cochlear nerve size by magnetic resonance imaging in elderly patients with sensorineural hearing loss. Radiol Med 2010;115:483-7.

9) Ho HG, Lin HC, Shu MT, Yang CC, Tsai HT. Effectiveness of intratympanic dexamethasone injection in sudden-deafness patients as salvage treatment. Laryngoscope 2004;114:1184-9.

10) Fortnum H, O'Neill C, Taylor R, Lenthall R, Nikolopoulos T, Lightfoot $\mathrm{G}$, et al. The role of magnetic resonance imaging in the identification of suspected acoustic neuroma: a systematic review of clinical and cost effectiveness and natural history. Health Technol Assess 2009;13:iii-iv, ix-xi, 1-154.

11) Lammers MJW, Young E, Fenton D, Lea J, Westerberg BD. The prognostic value and pathophysiologic significance of three-dimensional fluid-attenuated inversion recovery (3D-FLAIR) magnetic resonance imaging in idiopathic sudden sensorineural hearing loss: a systematic review and meta-analysis. Clin Otolaryngol 2019;44: 1017-25.

12) Jaryszak EM, Patel NA, Camp M, Mancuso AA, Antonelli PJ. Cochlear nerve diameter in normal hearing ears using high-resolution magnetic resonance imaging. Laryngoscope 2009;119:2042-5.

13) Lee JW, Park YA, Park SM, Kong TH, Park SY, Bong JP, et al. Clinical features and prognosis of sudden sensorineural hearing loss secondary to intralabyrinthine hemorrhage. J Audiol Otol 2016;20:31-5.

14) Li J, Wang M, Sun L, Zhao H, Song G, Tian J, et al. The correlation analysis of intralabyrinthine haemorrhage magnetic resonance imaging with hearing loss and prognosis: a retrospective analysis of 207 cases. Clin Otolaryngol 2019;44:1096-100. 